\title{
MOISTURE RELATIONS OF TEXTILE FIBERS AT ELEVATED TEMPERATURES
}

\author{
By James G. Wiegerink ${ }^{1}$
}

\section{ABSTRACT}

The moisture contents of 10 kinds of textile fibers in the form of specially prepared yarns were determined when the fibers were in equilibrium with air for a series of relative humidities and temperatures. Data were obtained for both "desorption" and "adsorption," the yarns being brought to equilibrium from a wet condition and a dry condition, respectively. The fibers studied were raw cotton, "purified" cotton, mercerized cotton, clothing wool, carpet wool, viscose rayon, cuprammonium rayon, raw silk, degummed silk, and cellulose acetate. The temperatures ranged from $96^{\circ}$ to $302^{\circ} \mathrm{F}$, and the relative humidities ranged from 5 to 90 percent for temperatures below $212^{\circ} \mathrm{F}$ and up to the maximum obtainable at atmospheric pressure above $212^{\circ} \mathrm{F}$.

The results are given in the form of graphs showing moisture content against relative humidity and also in the form of graphs in which the logarithms of the moisture contents at given relative humidities are plotted against the reciprocals of the corresponding absolute temperatures. The last show straight-line relationships with changes in the slopes of the lines between $200^{\circ}$ and $220^{\circ} \mathrm{F}$.

\section{CONTENTS}

I. Introduction

II. Materials

III. Procedure

IV. "Dry" weight of the fibers

V. Results and conclusions

\section{INTRODUCTION}

As part of a program of investigation of the drying of textiles, the moisture contents of 10 kinds of textile fibers were determined when the fibers were in equilibrium with air at different relative humidities and temperatures ranging from $96^{\circ}$ to $302^{\circ} \mathrm{F}$. Equilibrium was approached from both the wet state (desorption) and from the dry state (adsorption). The desorption data, of importance in the drying of textiles, include measurements at nine temperatures and at as many relative humidity values at each temperature as were considered necessary or expedient. Since an analysis of the desorption data revealed a linear relationship between the logarithm of the moisture content and the reciprocal of the absolute temperature from which intermediate values could be obtained, the adsorption data, of importance in the reconditioning of textiles, were obtained for only five temperatures. Below the boiling point of water at atmospheric

\footnotetext{
1 Research Associate at the National Bureau of Standards, representing the United States Institute for Textile Research. The establishment of this research associateship was made possible by funds contributed by 57 individual manufacturers.
} 


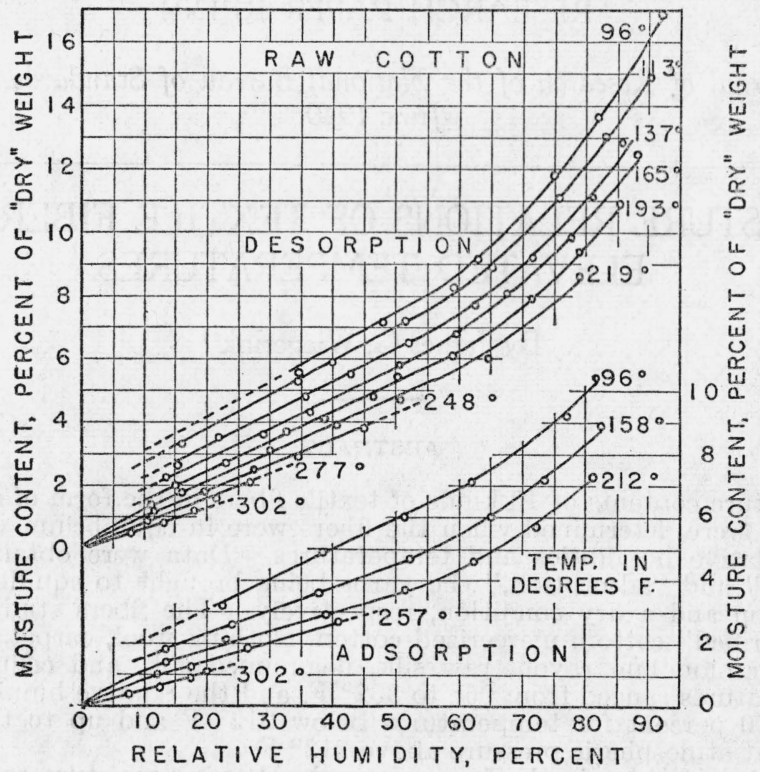

FIGURE 1.-Equilibrium moisture content of raw cotton yarn at different relative humidities and temperatures.

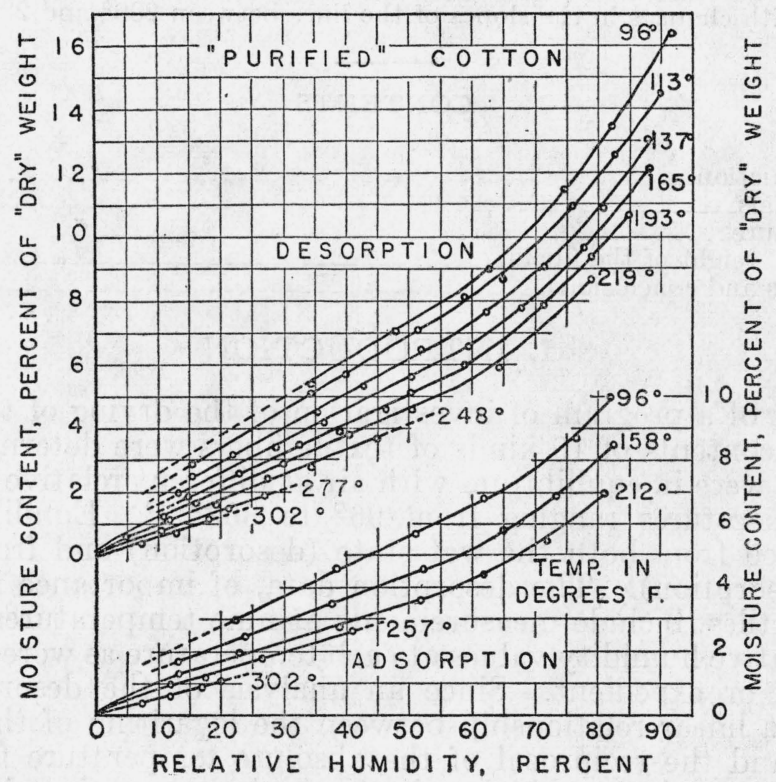

FIGURE 2.-Equilibrium moisture content of "purified" cotton yarn at different relative humidities and temperatures. 


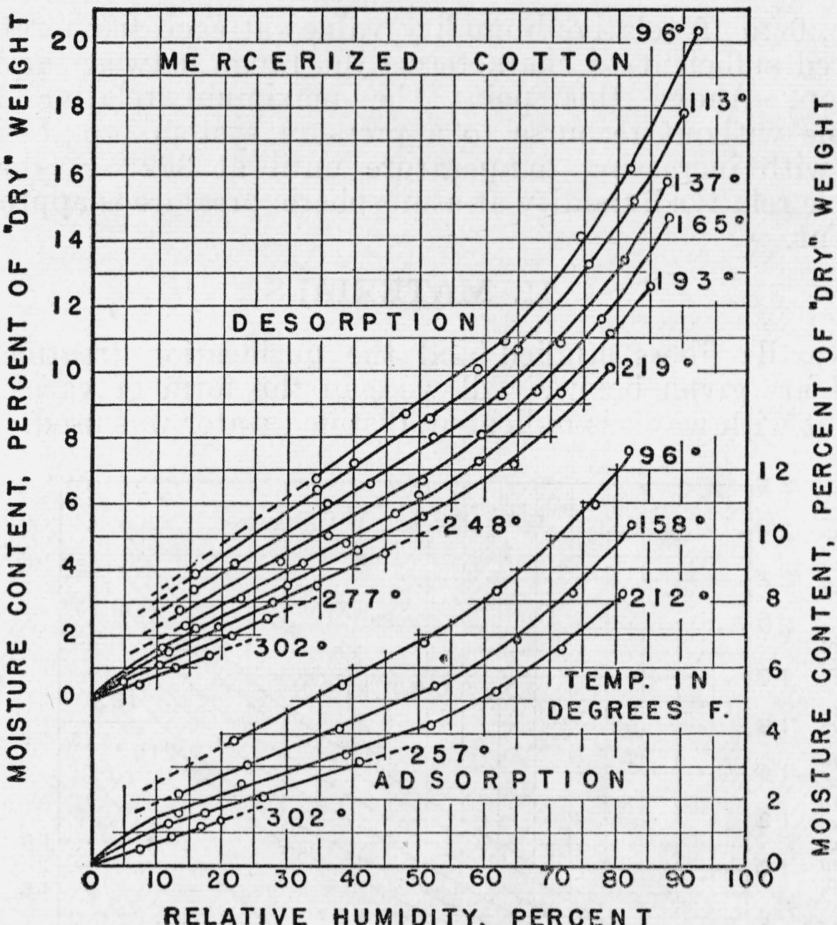

FIGURE 3.-Equilibrium moisture content of mercerized cotton yarn at different relative humidities and temperatures.

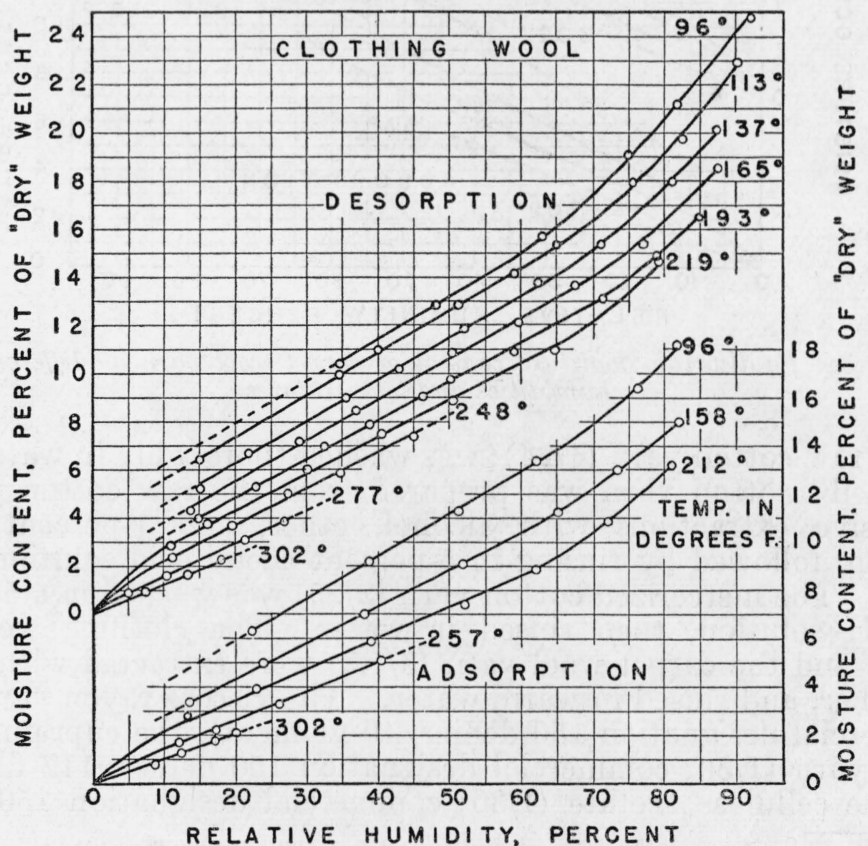

FIGURE 4.-Equilibrium moisture content of clothing wool yarn at different relative humidities and temperatures. 
pressure, 6 to 10 relative-humidity values at each temperature were considered sufficient to characterize the range between dryness and saturation. Above this point the maximum relative humidity attainable without recourse to a pressure system progressively decreases with increasing temperature until at $302^{\circ} \mathrm{F}$, the highest attainable relative humidity at atmospheric pressure is approximately 20 percent.

\section{MATERIALS}

The textile fibers studied and the purification treatments they received are given below. All were in the form of yarns. Where treatment with water is indicated, distilled water was used.

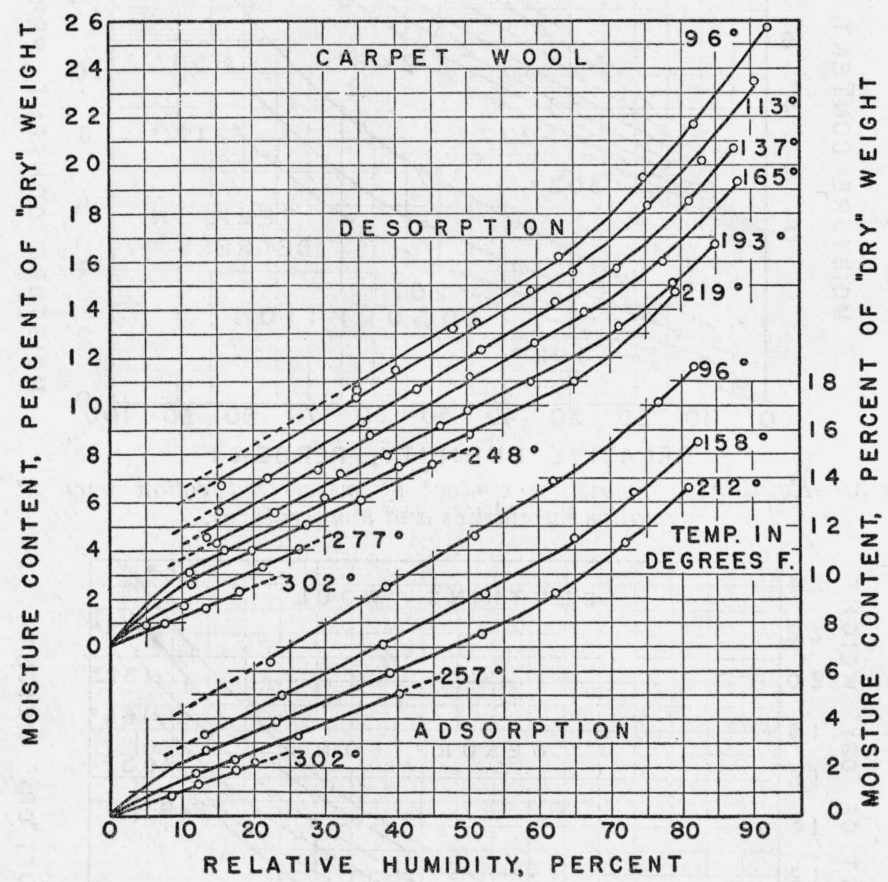

FIGURE 5.-Equilibrium moisture content of carpet wool yarn at different relative humidities and temperatures.

The raw cotton yarn $(1 / 24)^{2}$ was washed thoroughly in water. The "purified" cotton yarn was prepared from the raw cotton yarn by successive extractions with alcohol, ether, and 1-percent $\mathrm{NaOH}$ solution, followed by rinsing in 5-percent acetic acid solution and in water. The mercerized cotton yarn $(2 / 25)$ was washed in a 5 -percent $\mathrm{NH}_{4} \mathrm{OH}$ solution, then rinsed in water. The clothing wool yarn $(2 / 2.3)$ and the carpet wool yarn (3/1.2) were extracted with alcohol and ether, and rinsed in warm water. The viscose rayon yarn $(1 / 30$; commercial designation 150 denier, 40 filament), the cuprammonium rayon yarn (1/30; commercial designation 150 denier, 112 filament), and the cellulose acetate $(1 / 30$; commercial designation 150 denier,

\footnotetext{
${ }^{2}$ The typp system for designating sizes of yarns is employed. The first number (1 in the case of the raw cotton yarn) indicates the number of units of which the yarn is composed, that is, the ply. The second number given ( 24 in the case of the raw cotton yarn) indicates the number of thousands of yards in 1 pound.
} 
46 filament) were washed in a warm 1-percent $\mathrm{NH}_{4} \mathrm{OH}$ solution, then rinsed in warm water. The raw silk yarn $(1 / 300$; commercial designation 13-15 denier) was washed in water at room temperature. The degummed silk was prepared from the raw silk yarn by degumming with an olive oil soap solution, followed by rinsing in warm water.

\section{PROCEDURE}

The equipment used for this investigation is described in detail in a previous paper. ${ }^{3}$ It consists of equipment for controlling the temperature and relative humidity of air, for passing a stream of this air

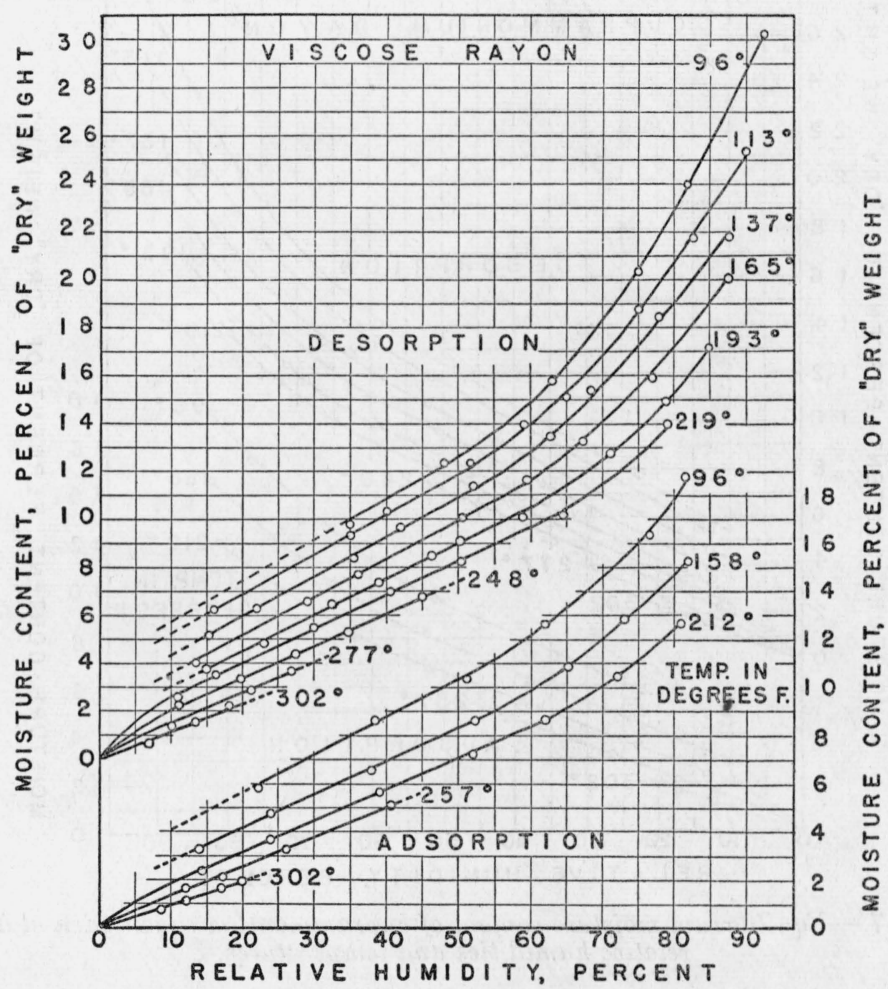

FIGURE 6.-Equilibrium moisture content of viscose rayon yarn at different relative humidities and temperatures.

through a working chamber at a known rate, for exposing the samples in this chamber to the air stream and weighing them in it conveniently and rapidly; and for sampling the air in the working chamber and accurately determining its relative humidity, by a gravimetric measurement of the moisture content. The temperature in the working chamber was maintained constant within $\pm 0.4^{\circ} \mathrm{F}$ and the relative humidity within \pm 1 -percent relative humidity throughout each test run. Some of the runs were 24 hours or more in duration.

${ }^{3}$ J. G. Wiegerink, Equipment for conditioning materials at const ant humidities and elevated temperatures, J. Research NBS 24, 639 (1940) RP 1303. 
The samples for the desorption experiments were conditioned by soaking them overnight in distilled water. Then the excess water was removed by gently squeezing the samples until the weights of squeezed yarns were about twice that of dry weights, and the samples were then placed in the working chamber at the temperature and the initial high humidity of the test condition. They were brought to equilibrium at a series of progressively decreasing relative-humidity values, weighings being made at suitable intervals until no change in weight was found over a period of approximately 12 hours. The

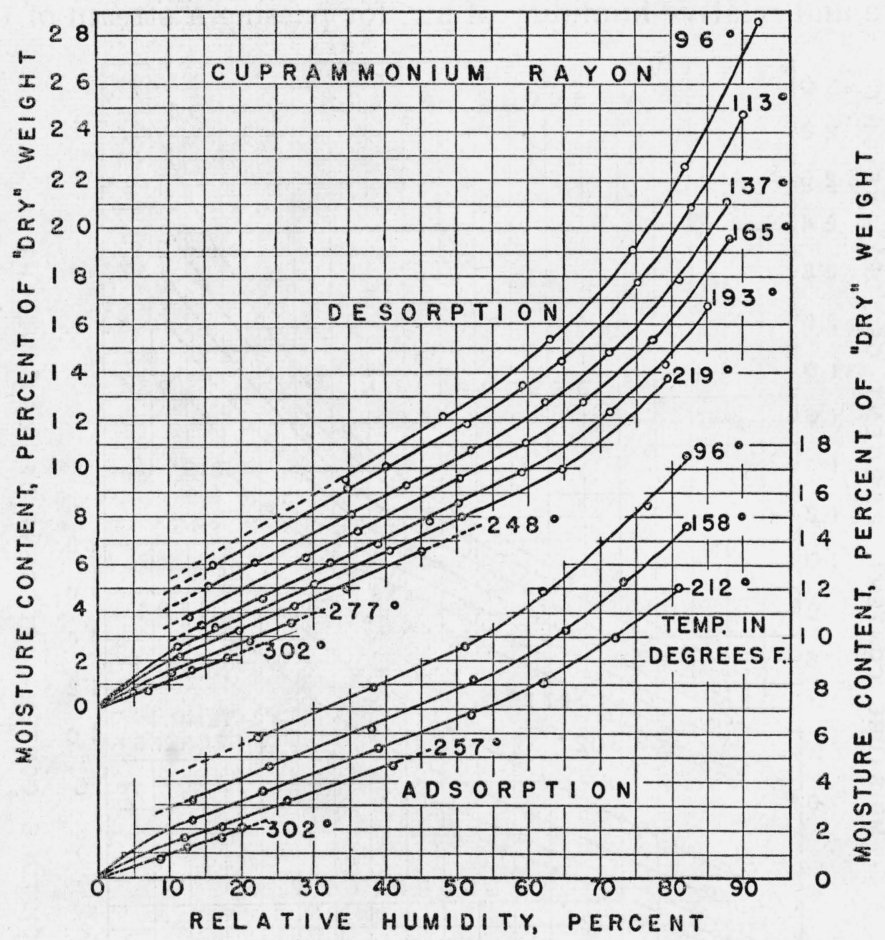

FIGURE 7.-Equilibrium moisture content of cuprammonium rayon yarn at different relative humidities and temperatures.

adsorption samples were conditioned by drying over $\mathrm{Mg}\left(\mathrm{ClO}_{4}\right)_{2} \cdot-$ $3 \mathrm{H}_{2} \mathrm{O}$ at $75^{\circ} \mathrm{F}$ for 2 weeks. They were then placed quickly in the working chamber and successively brought to equilibrium at a series of increasing relative-humidity values.

\section{IV. "DRY" WEIGHT OF THE FIBERS}

The "dry" weights of the samples used in the desorption experiments were obtained after the completion of the series of experiments by heating the samples in an air oven at $212^{\circ} \mathrm{F}$ overnight.

The "dry" weights of the samples used for the adsorption experiments were obtained prior to the experiments in the working chamber. 
Since heating in an air oven at $212^{\circ} \mathrm{F}$ might have rendered the samples unfit for tests at lower temperatures, they were dried by suspending them from a wire passing through narrow glass tubing in the stopper of the bottle containing the drier. This made it possible to weigh the sample without removing it from the air over the drier. The samples required 2 weeks to reach constant weight.

The samples thus dried had a "dry" weight a little lower than those dried in the air oven at $212^{\circ} \mathrm{F}$ (the relative humidity in the oven was from 1.5 to 2.0 percent). The differences for the various

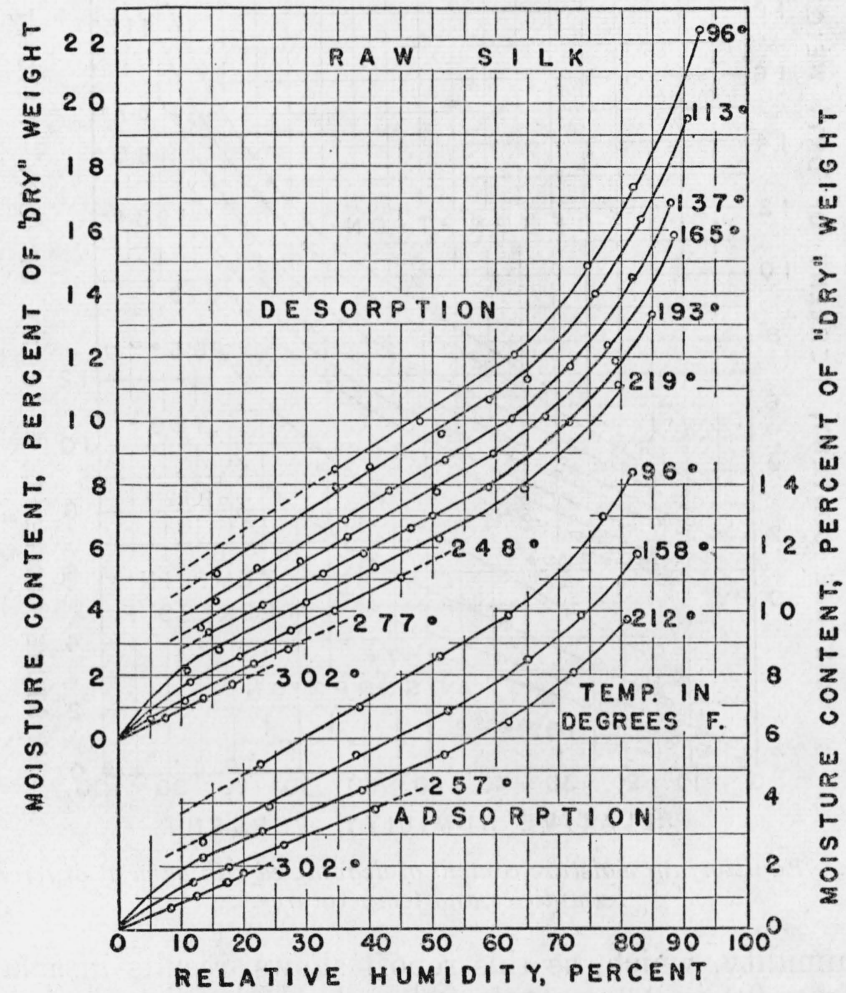

FIGURE 8.-Equilibrium moisture content of raw silk yarn at different relative humidities and temperatures.

fibers averaged:For raw " and "purified" cotton, 0.15 percent; for clothing wool, carpet wool, viscose rayon, and cuprammonium rayon, 0.30 percent; for mercerized cotton and raw silk, 0.20 percent; and for degummed silk and acetate, 0.10 percent. All desorption data were corrected by these amounts. Thus the moisture contents given in this report are based upon the weight of the material as dried over the chemical drier. They are expressed as percentage of the dry weight of the material. In the textile industry this is termed "regain."

No attempt is made here to differentiate between "free" and "bound" water in textiles or to discuss the merits of such a differen- 
tiation. It is realized that the term "dry" may have several connotations, varying from the exceedingly dry condition achieved by keeping materials in sealed tubes over an efficient drying agent for periods of several years, to a superficial drying, commonly described as "bonedry", achieved by drying in an air oven maintained at or slightly above the boiling point of water, but open to the prevailing atmos-

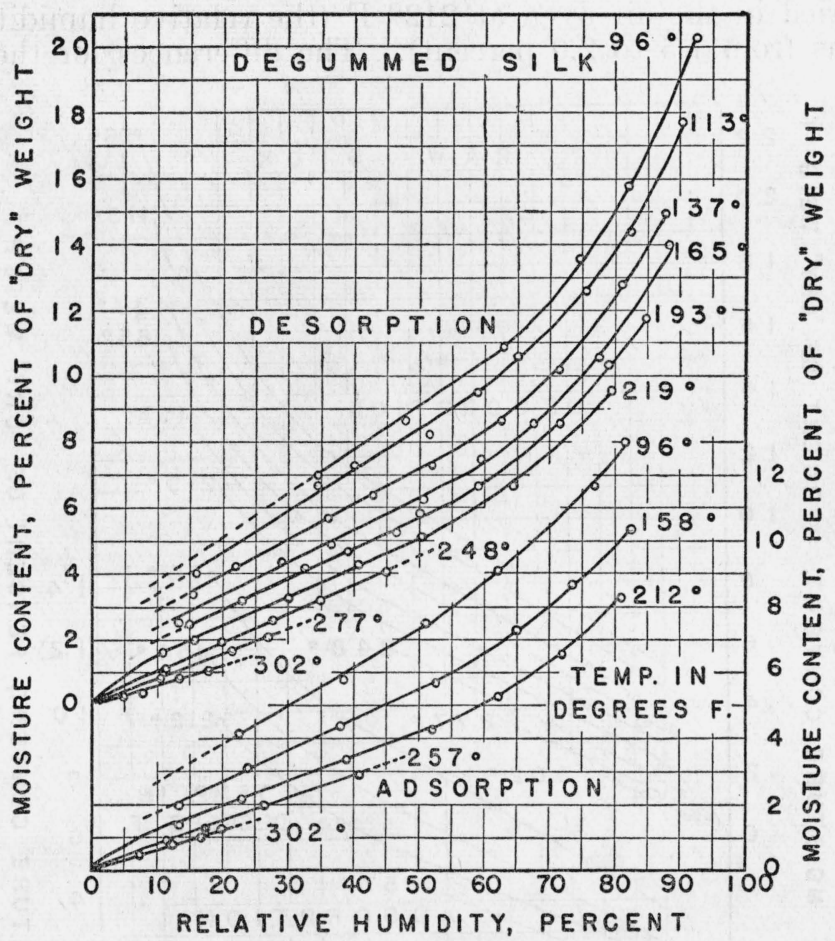

FIGURE 9.-Equilibrium moisture content of degummed silk yarn at different relative humidities and temperatures.

pheric humidity, which, as this report shows, results in a failure to remove from 0.1 to 0.3 percent of the adsorbed moisture.

\section{RESULTS AND CONCLUSIONS}

In the figures 1 to 10 are curves of the familiar form expressing the relation between percentage moisture content and percentage relative humidity for each temperature and each material. Each point on the curves is the average of two determinations. The broken lines show extrapolated values. This extrapolation is quite justifiable, since the relation between percentage moisture content and percentage relative humidity is linear in the range 10- to 60percent relative humidity.

These curves indicate the limits in moisture content which may be expected in any of the textile fibers if the atmosphere in contact 
with the fiber during drying is maintained at any desired temperature and relative humidity. The "desorption" curve for the given temperature is to be used if the fiber is initially wet, and the "adsorption" curve is to be used if the fiber is initially dry. It should be emphasized that the curves apply, of course, only to the samples examined. Further work is necessary to determine what variation

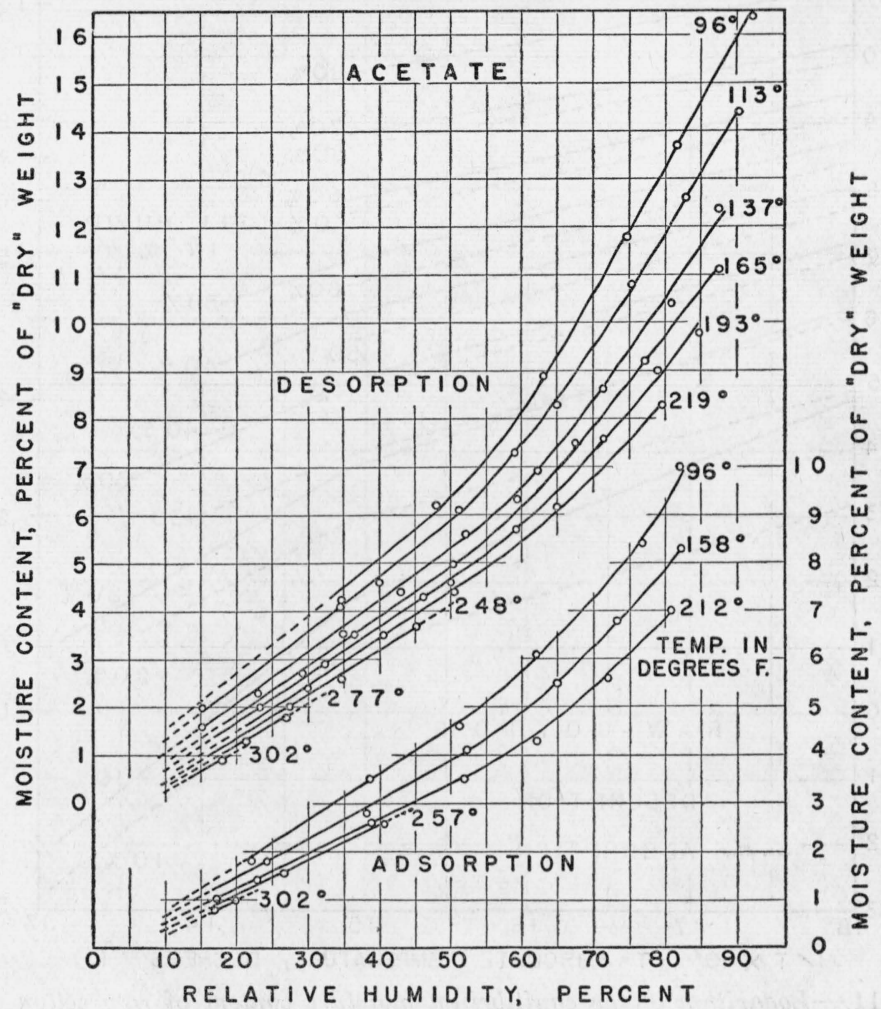

FIGURE 10.-Equilibrium moisture content of cellulose acetate yarn at different relative humidities and temperatures.

is to be expected in different lots of the same fiber, or when the fibers contain impurities, such as oils, sizing materials, and dyes.

A study of these data revealed that if the logarithms of the moisture contents at any fixed relative humidity are plotted against the reciprocals of the corresponding absolute temperatures $(1 / T)$, straightline relationships are obtained with changes in the slopes of the lines between $200^{\circ}$ and $220^{\circ} \mathrm{F}$. The slope of the "desorption" line thus obtained gives the rate of decrease in equilibrium moisture content of a textile, with increasing temperature, at constant relative humidity. The slope of the "adsorption" line gives the rate of increase in equilibrium moisture content, with decreasing temperature, at constant relative humidity. Thus a series of such linear relations may be prepared, one for each relative humidity, from which it is possible to ascertain the moisture content for any humidity at any tempera- 
ture within the range investigated. Since Urquhart and Williams ${ }^{4}$ obtained similar data on cotton, including temperatures as low as $50^{\circ} \mathrm{F}$, and since the relation holds for their data as well as for those

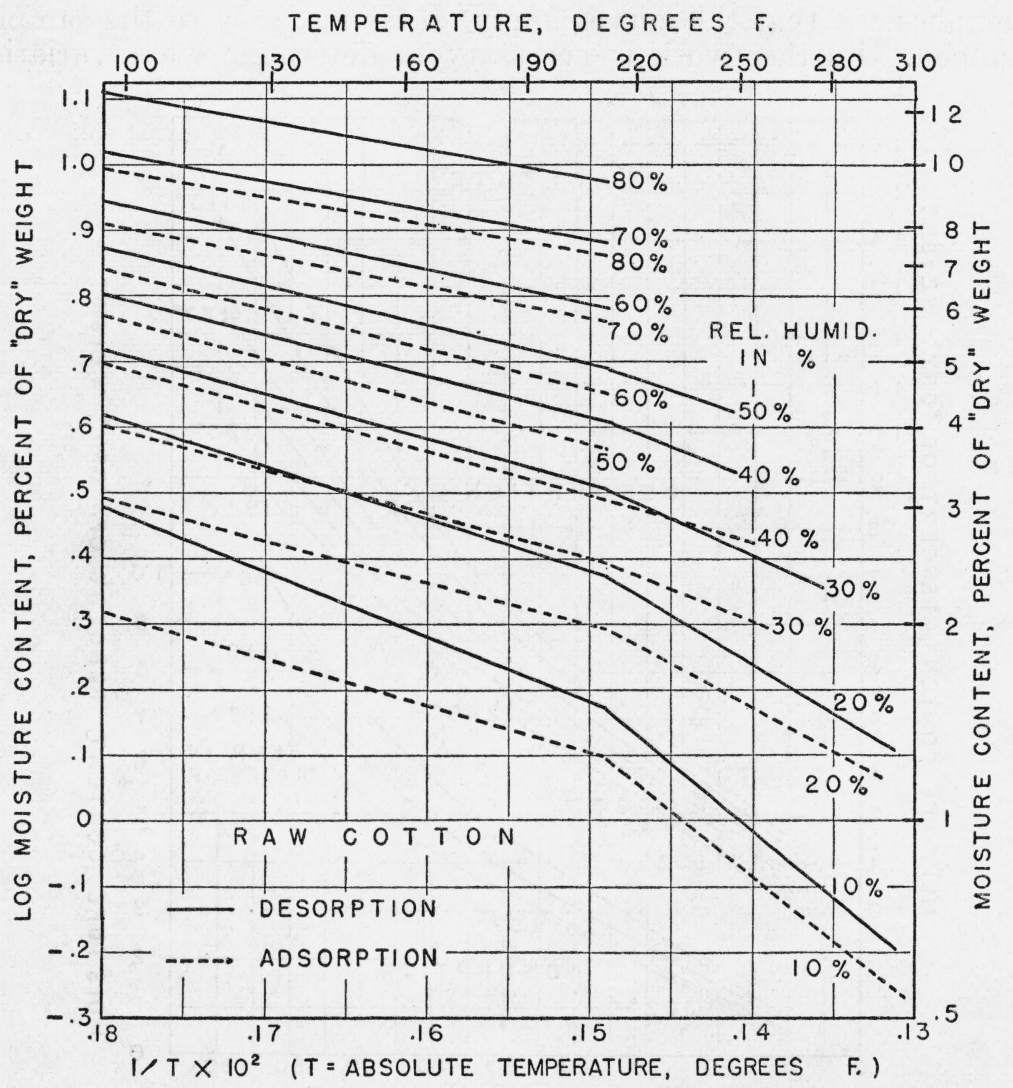

FIGURE 11.-Logarithm of the equilibrium moisture content of raw cotton yarn in relation to the reciprocal of the absolute temperature $(1 / T)$.

presented here, it seems reasonable to assume that the linear relation may be extrapolated down to temperatures somewhat below room temperature, if desired.

4 Urquhart and Williams, J. Text. Inst. 15, T559-572 (1924). 
Figures 11 to 20 show these relations for humidities at 10 -percent intervals between 10- and 80-percent relative humidity. The unbroken lines give this relationship for the desorption data, the broken

TEMPERATURE, DEGREES F.

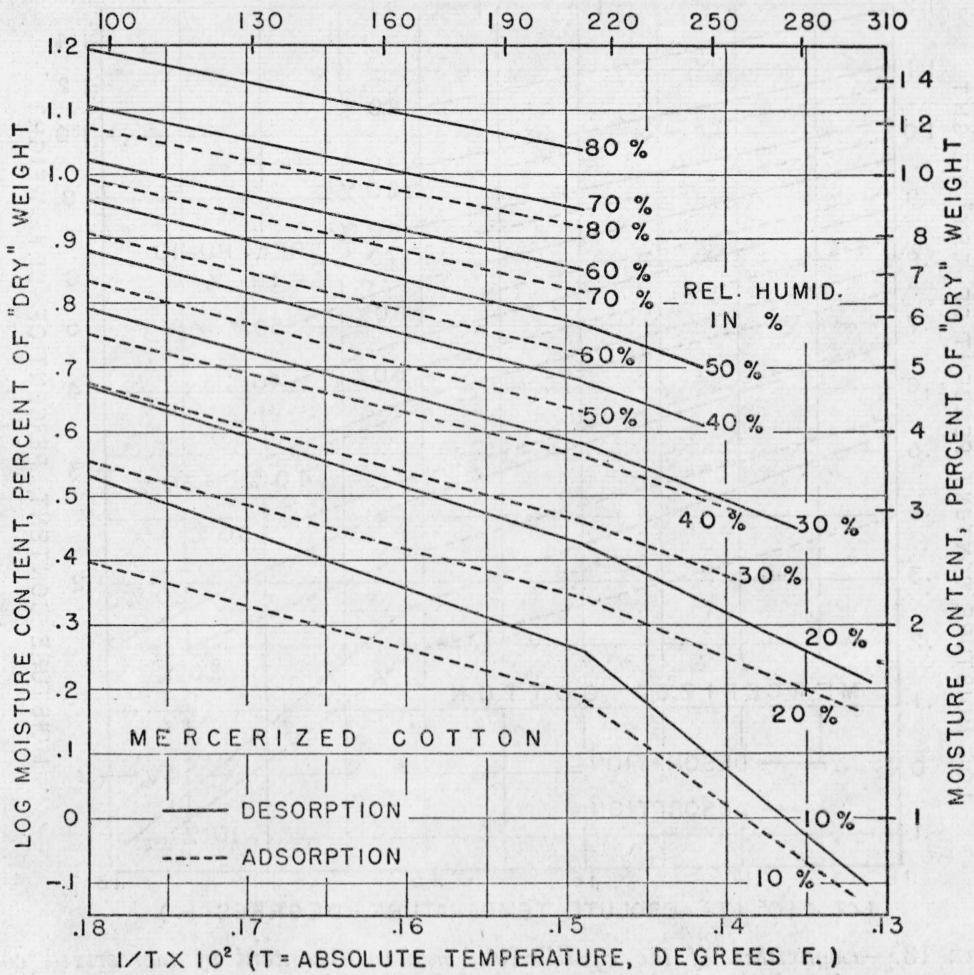

FIgURE 12.-Logarithm of the equilibrium moisture content of "purified" cotton yarn in relation to the reciprocal of the absolute temperature $(1 / T)$.

lines for the adsorption data. The data for these graphs were taken from the curves given in figures 1 to 10 and are not represented as points on these graphs in order to avoid confusion between the ad- 
sorption and desorption curves. An average example of how closely the logarithms of the moisture contents follow a linear relationship

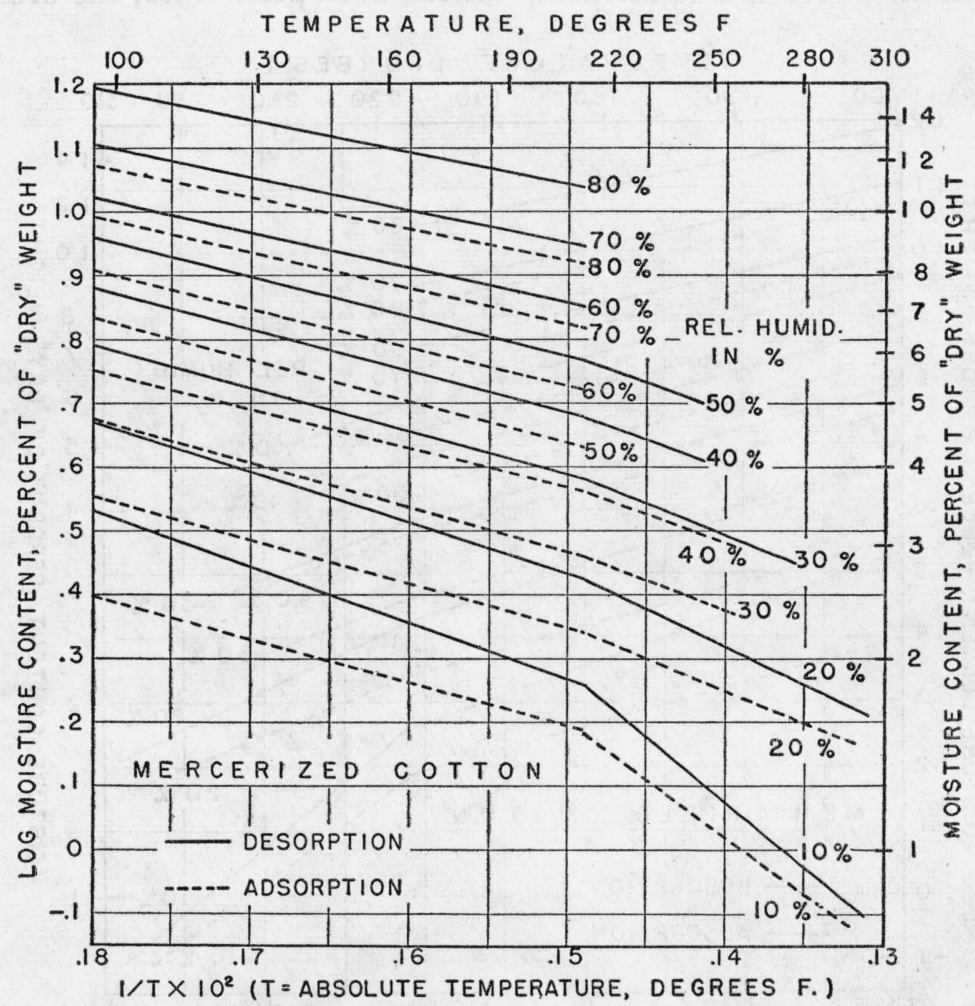

FIGURE 13.-Logarithm of the equilibrium moisture content of mercerized cotton yarn in relation to the reciprocal of the absolute temperature $(1 / T)$.

with the reciprocals of the corresponding absolute temperatures is shown by figure 21, which gives the desorption data for viscose rayon. 
Examination of figures 11 to 20 shows that the linear relationships obtained give changes in the slopes of the lines between $200^{\circ}$ and

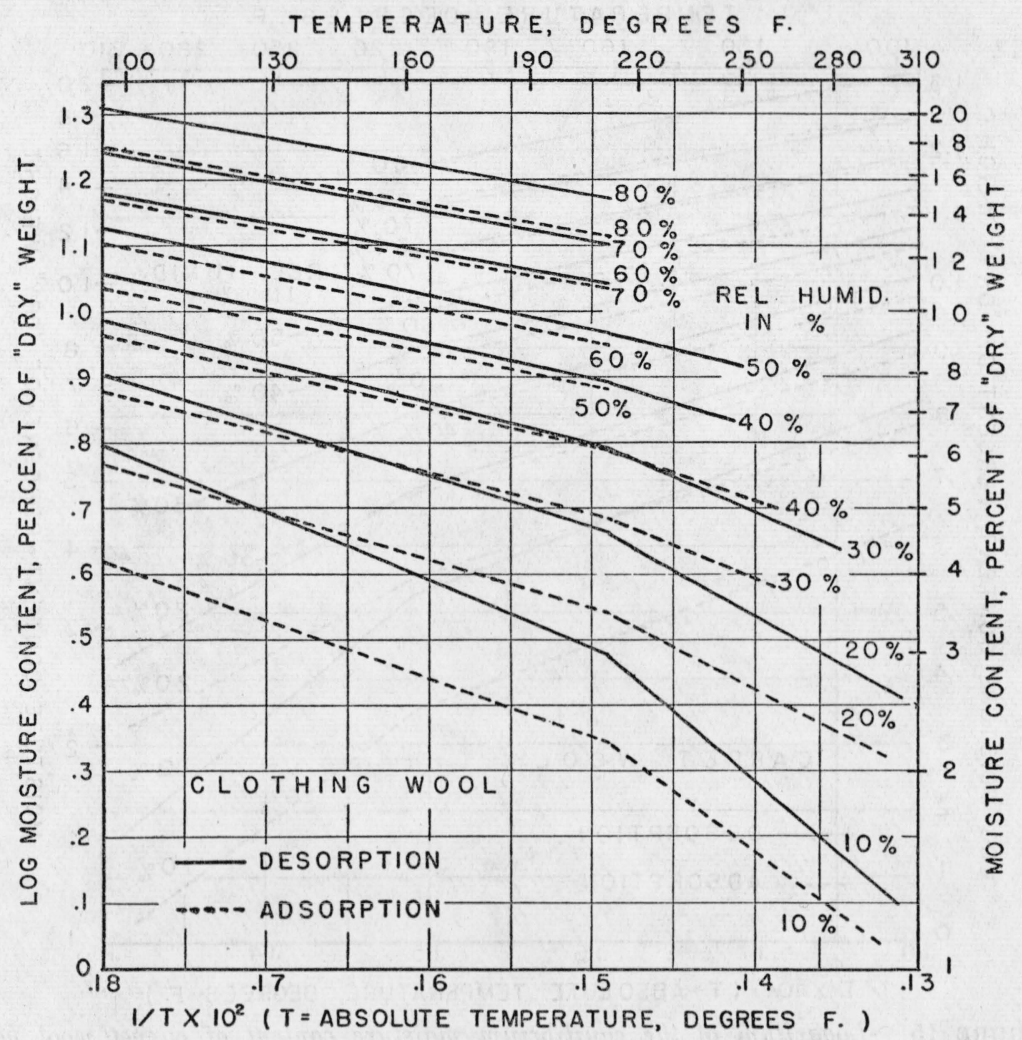

FIGURE 14.-Logarithm of the equilibrium moisture content of clothing wool yarn in relation to the reciprocal of the absolute temperature $(1 / T)$.

$220^{\circ} \mathrm{F}$. The data are represented for convenience by straight lines but could be better fitted by a smooth curve. The bending of these 
curves is of significance in connection with heats of swelling, but the theoretical analysis has not yet been carried out.

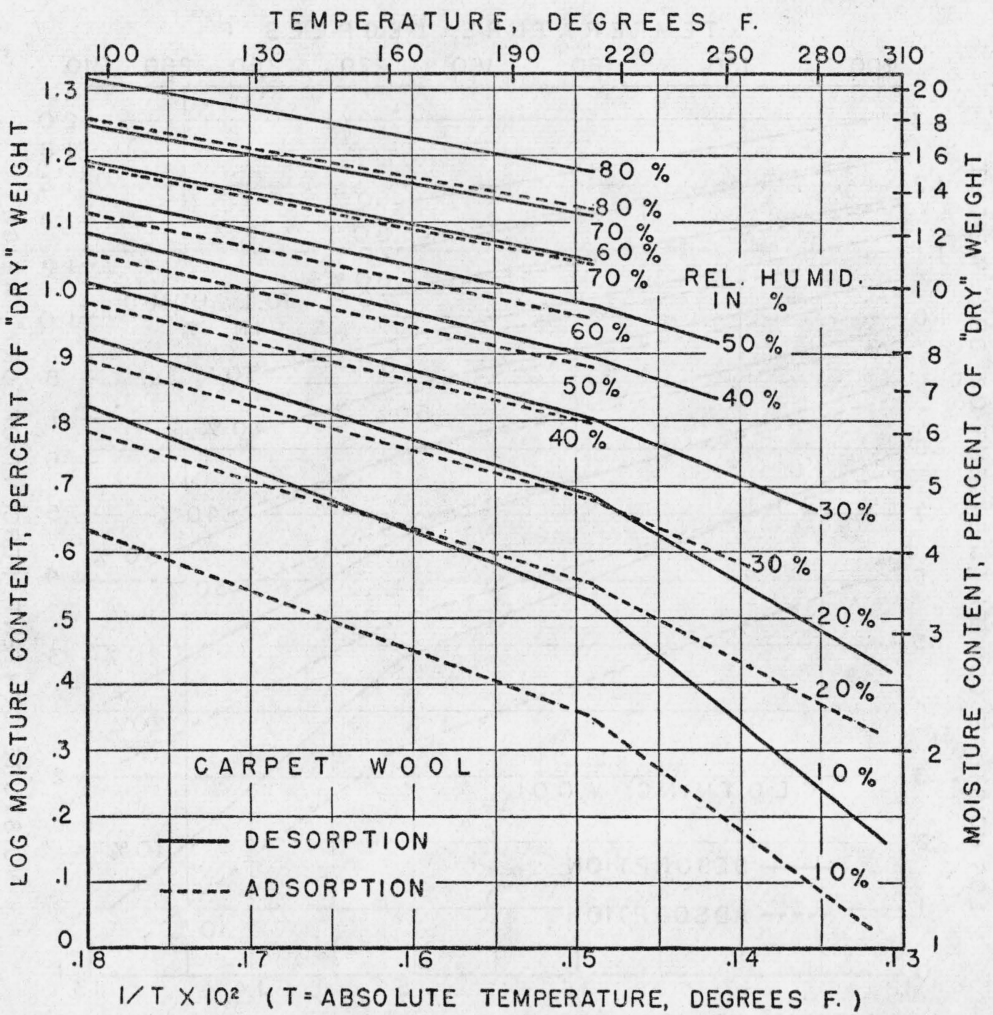

FIGURE 15.-Logarithm of the equilibrium moisture content of carpet wool yarn in relation to the reciprocal of the absolute temperature $(1 / T)$.

Certain generalizations may be made for all the textiles examined. Above 30-percent relative humidity the slope of the linear relation 
changes very little with increasing humidity, in the range between $100^{\circ}$ to $200^{\circ} \mathrm{F}$. Below 30-percent relative humidity the slope in-

TEMPERATURE, DEGREES F.

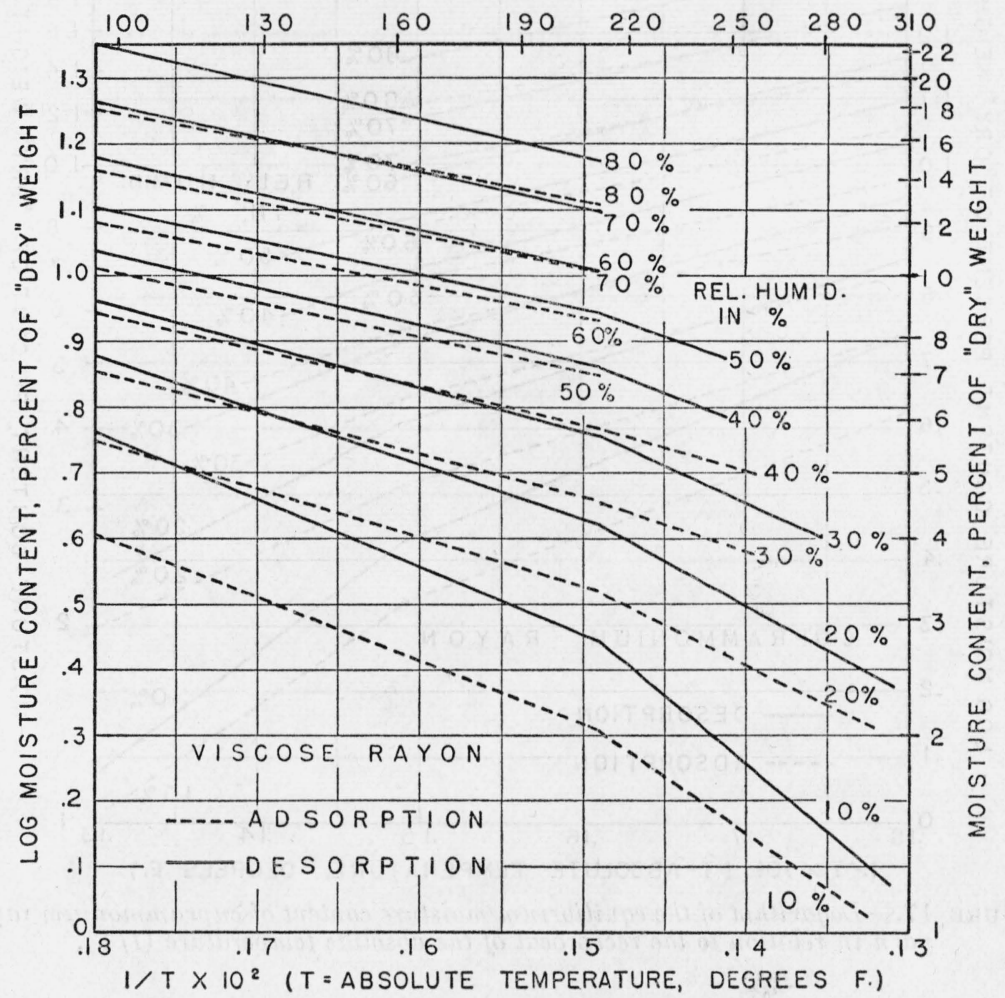

FIGURE 16.-Logarithm of the equilibrium moisture content of viscose rayon yarn in relation to the reciprocal of the absolute temperature $(1 / T)$.

creases appreciably with decreasing humidity. Above $200^{\circ} \mathrm{F}$ the slopes change for all relative humidities investigated up to 50 percent. 
TEMPERATURE, DEGREES F.

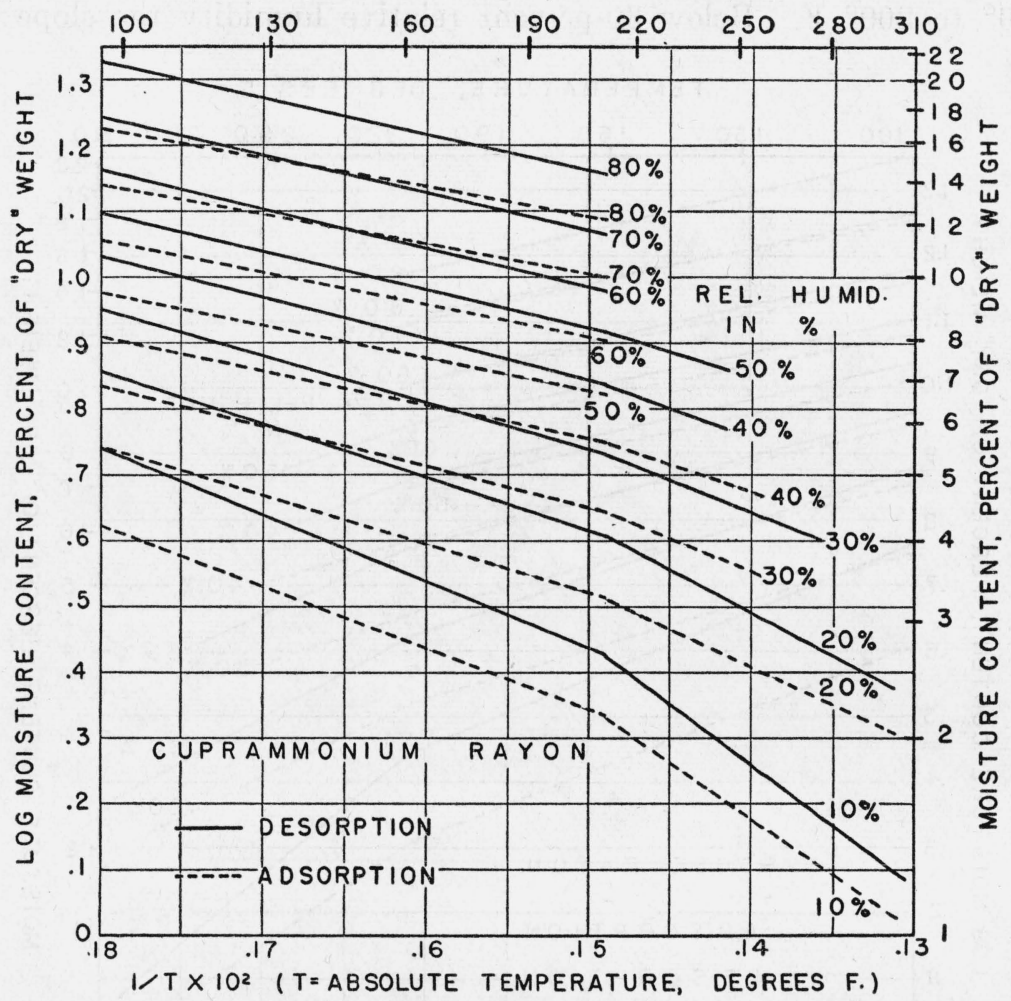

FIGURE 17.-Logarithm of the equilibrium moisture content of cuprammonium rayon yarn in relation to the reciprocal of the absolute temperature $(1 / T)$. 
TEMPERATURE, DEGREES F.

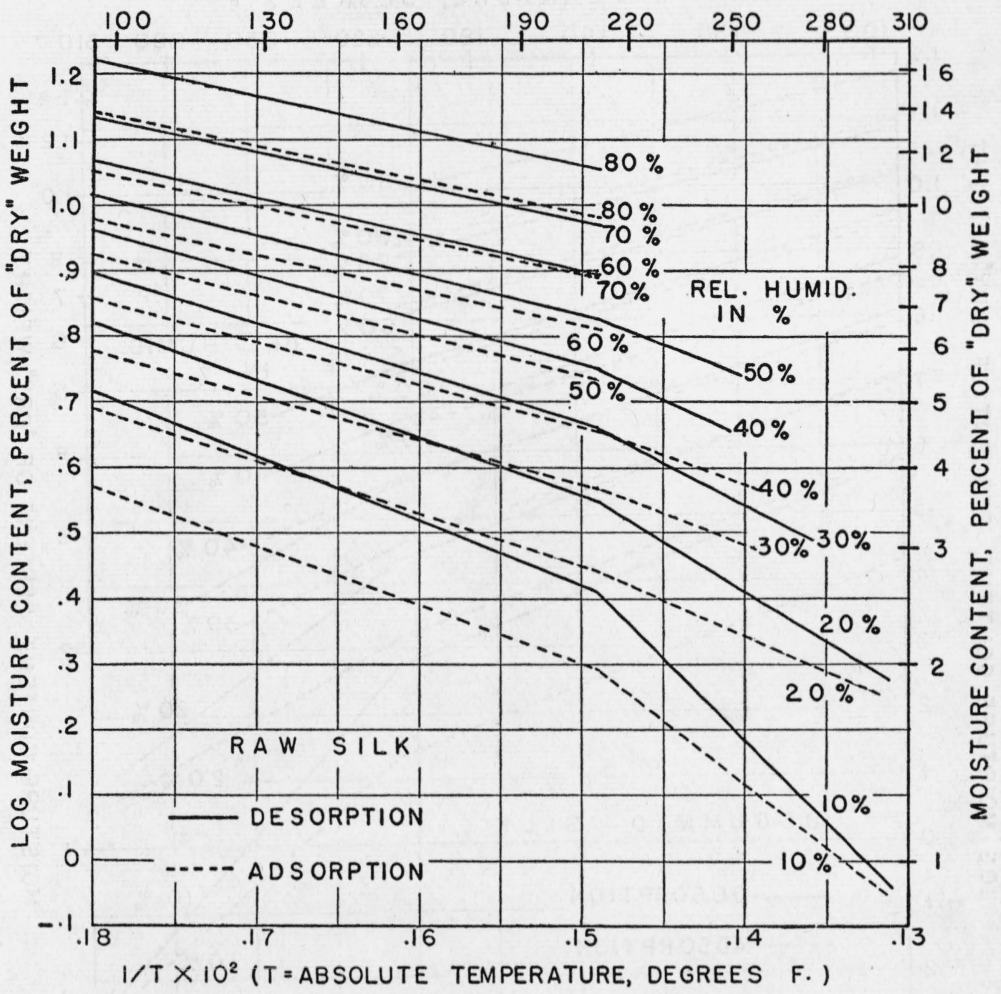

FIGURE 18.-Logarithm of the equilibrium moisture content of raw silk yarn in relation to the reciprocal of the absolute temperature $(1 / T)$. 
TEMPERATURE, DEGREES F.

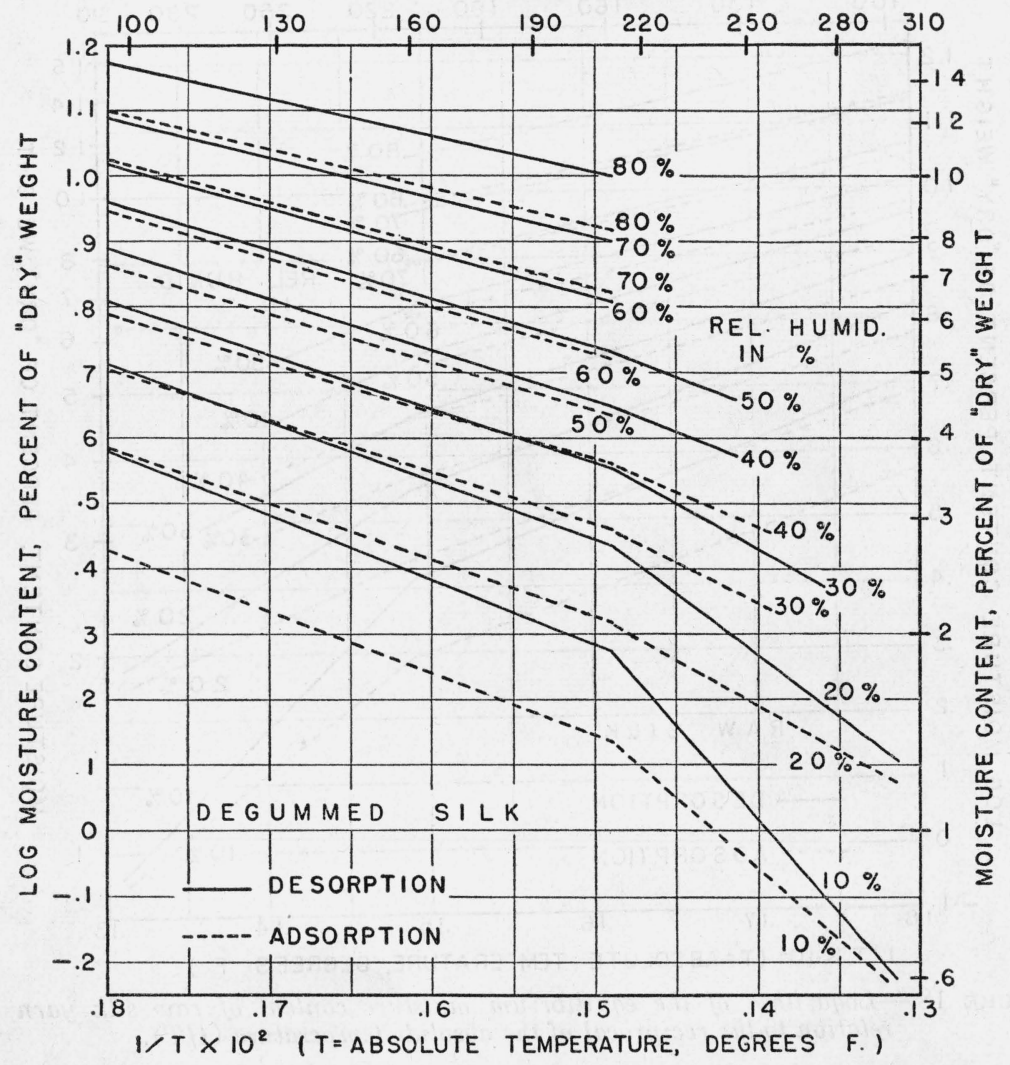

FIgURE 19.-Logarithm of the equilibrium moisture content of degummed silk yarn in relation to the reciprocal of the absolute temperature $(1 / T)$. 


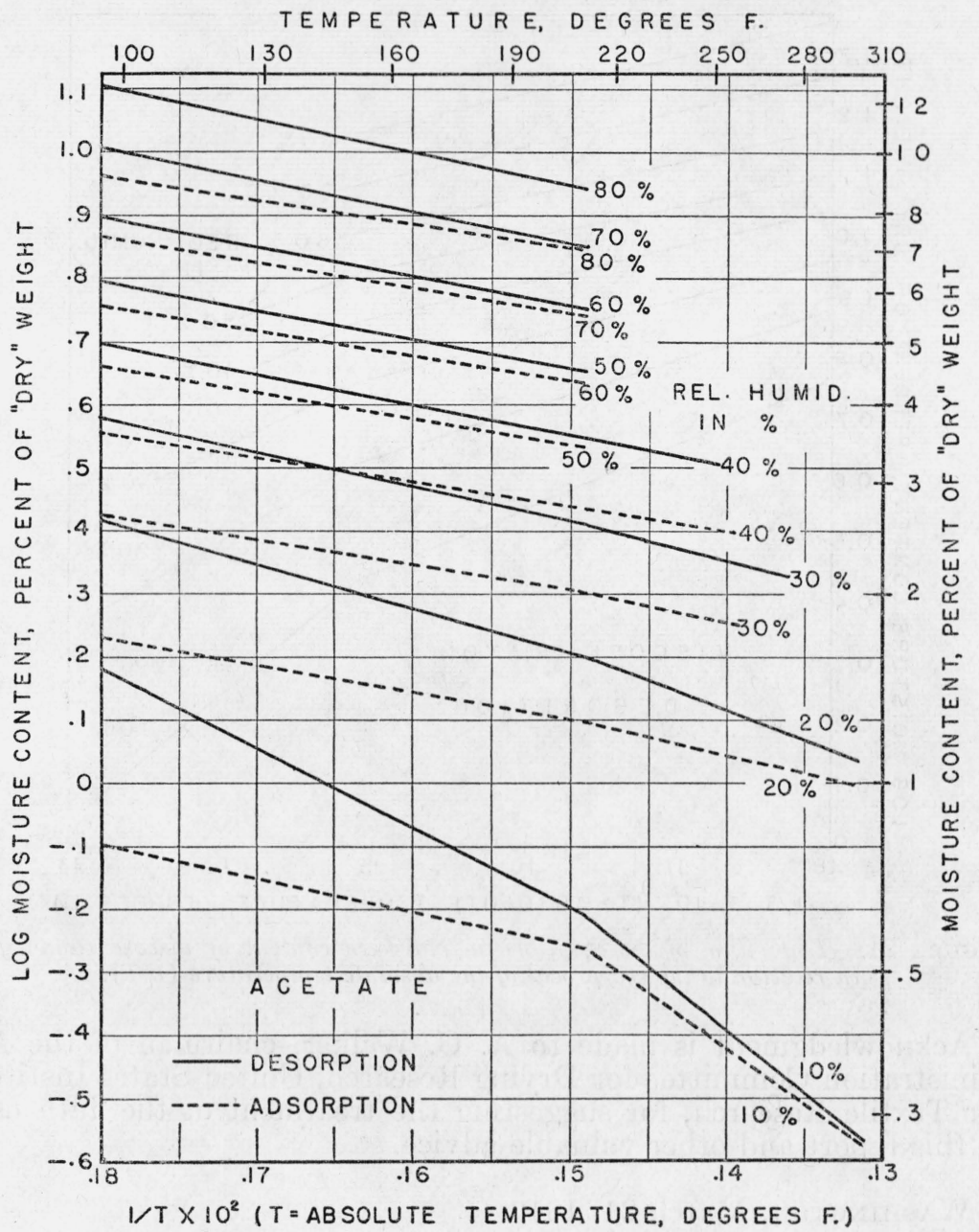

FIGURE 20.-Logarithm of the equilibrium moisture content of cellulose acetate yarn in relation to the reciprocal of the absolute temperature $(1 / T)$. 


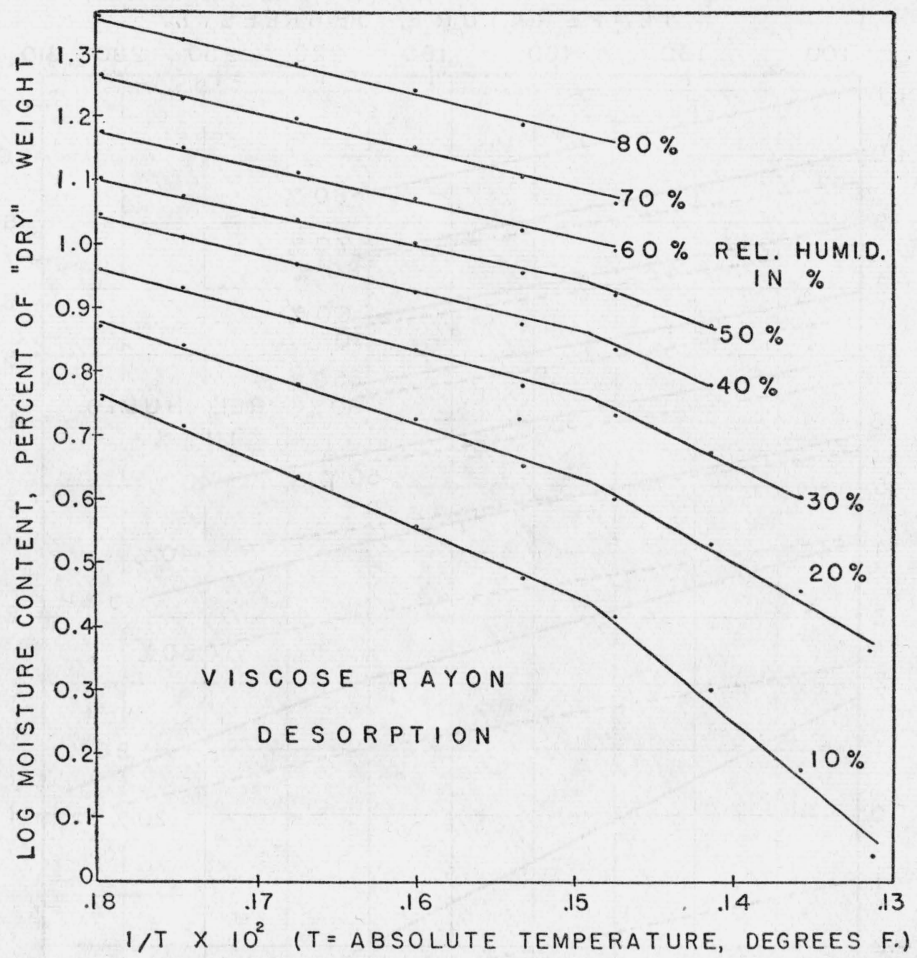

FIGURE 21.-Logarithm of the equilibrium moisiure content of viscose rayon yarn in relation to the reciprocal of the absolute temperature $(1 / T)$.

Acknowledgment is made to A. C. Walker, chairman of the Administration Committee for Drying Research, United States Institute for Textile Research, for suggesting the treatment of the data used in this report and other valuable advice.

WASHington, March 21, 1940. 